\title{
UTILIZAÇÃO DA ESCALA DE BRADEN COMO FERRAMENTA PARA AVALIAÇÃO DE FATORES DE RISCO DE LESÃO POR PRESSÃO EM IDOSOS
}

DOI: 10.48140/digitaleditora.2021.005.12

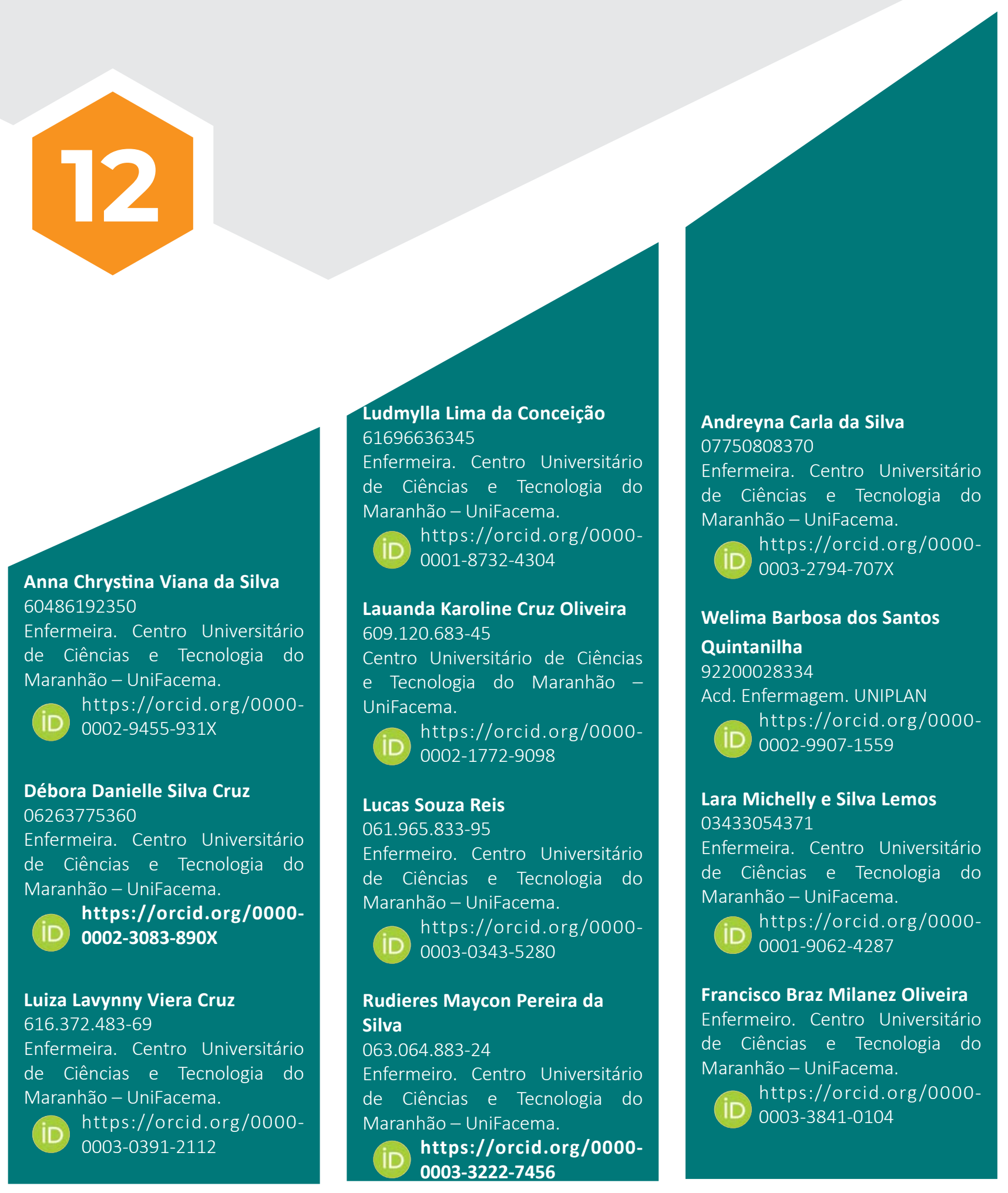




\section{UTILIZAÇÃO DA ESCALA DE BRADEN COMO FERRAMENTA PARA AVALIAÇÃO DE FATORES DE RISCO DE LESÃO POR PRESSÃO EM IDOSOS}

DOI: 10.48140/digitaleditora.2021.005.12

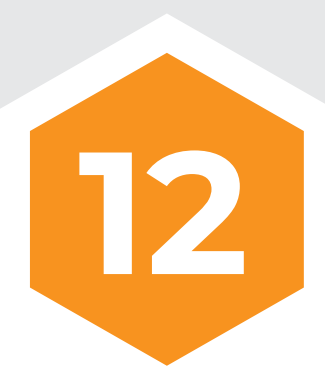

RESUMO

Recebido em: 30/11/2020

Aprovado em: 10/12/2020

Conflito de Interesse: não

Suporte Financeiro: não houve
Objetivos: Avaliar na produção científica a eficácia da escala de Braden para avaliação de fatores de risco de lesão por pressão em idosos.

Metodologia: Trata-se de uma pesquisa bibliográfica do tipo revisão integrativa da literatura na qual foi utilizada para a geração da questão norteadora desta revisão integrativa da literatura: "Quais evidências científicas apontam a eficácia da utilização da escala de Braden como ferramenta de avaliação de fatores de risco de lesão por pressão em idosos?".

Resultados: Foram incluídos quatro estudos nesta revisão que avaliaram a eficácia da escala de Braden na detecção precoce de lesões por pressão. A maioria das publicações foram concentradas no ano de 2014. Elencou-se um conjunto de duas intervenções de enfermagem necessárias para a prevenção de lesões por pressão (LPP) através da escala de Braden são elas: executar análise dos fatores de risco para lesões por pressão com a escala de Braden e aplicar reforço aos resultados da escala de braden com a escala de EMINA. Conclusão: A escala de Braden foi identificada como um instrumento confiável e efetivo para prever os pacientes com maior risco de desenvolver leões por pressão. 


\section{USE OF THE BRADEN SCALE AS A TOOL FOR THE EVALUATION OF RISK FACTORS FOR PRESSURE INJURY IN THE ELDERLY}

DOI: 10.48140/digitaleditora.2020.001.12

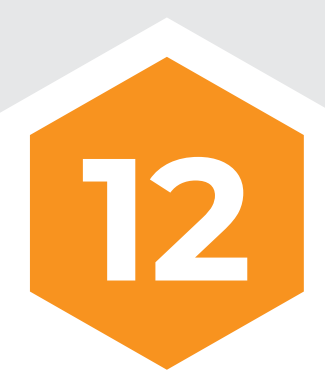

ABSTRACT

Recebido em: 30/11/2020

Aprovado em: 10/12/2020

Conflito de Interesse: não

Suporte Financeiro: não houve
Objectives: To evaluate the effectiveness of the Braden scale in evaluating risk factors for pressure injury in the elderly.

Methodology: this is a bibliographical research of the type integrative review of the literature in which it was used to generate the guiding question of this integrative review of the literature: "What scientific evidence points to the effectiveness of the use of the Braden scale as a factor evaluation tool risk of pressure injury in the elderly? ".

Results: Four studies were included in this review that evaluated the effectiveness of the Braden scale in the early detection of pressure lesions. Most of the publications were concentrated in the year 2014. A set of two nursing interventions required for the prevention of pressure lesions (LPP) through the Braden scale were identified: they performed analysis of the risk factors for pressure lesions with the Braden scale and apply reinforcement to the results of the braden scale with the EMINA scale.

Conclusion: The Braden scale was identified as a reliable and effective instrument to predict patients at higher risk of developing pressure lions. 


\section{USO DE LA ESCALA DE BRADEN COMO HERRAMIENTA PARA LA EVALUACIÓN DE FACTORES DE RIESGO DE LESIÓN POR PRESIÓN EN ANCIANOS}

DOI: 10.48140/digitaleditora.2020.001.12

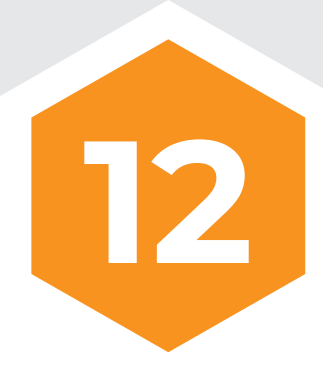

\section{RESUMEN}

Recebido em: 30/11/2020

Aprovado em: 10/12/2020

Conflito de Interesse: não

Suporte Financeiro: não houve
Objectives: Evaluar en la producción científica la eficacia de la escala de Braden para la evaluación de factores de riesgo de lesión por presión en ancianos.

Metodología: se trata de una investigación bibliográfica del tipo revisión integrativa de la literatura en la que fue utilizada para la generación de la cuestión orientadora de esta revisión integrativa de la literatura: "¿Cuáles evidencias científicas apuntan la eficacia de la utilización de la escala de Braden como herramienta de evaluación de factores de riesgo de lesión por presión en ancianos? ".

Resultados: se incluyeron cuatro estudios en esta revisión que evaluaron la eficacia de la escala de Braden en la detección precoz de lesiones por presión. La mayoría de las publicaciones se concentró en el año 2014. Se estableció un conjunto de dos intervenciones de enfermería necesarias para la prevención de lesiones a presión a través de la escala de Braden son: realizar análisis de los factores de riesgo para lesiones por presión con la escala de Braden y aplicar refuerzo a los resultados de la escala de braden con la escala de EMINA.

Conclusión: la escala de Braden fue identificada como un instrumento confiable y efectivo para predecir a los pacientes con mayor riesgo de desarrollar leones por presión. 


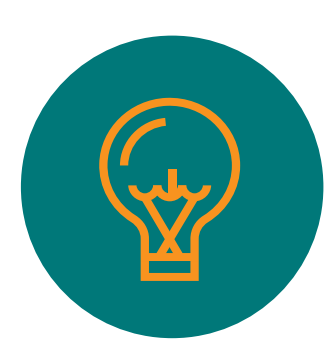

\section{INTRODUÇÃO}

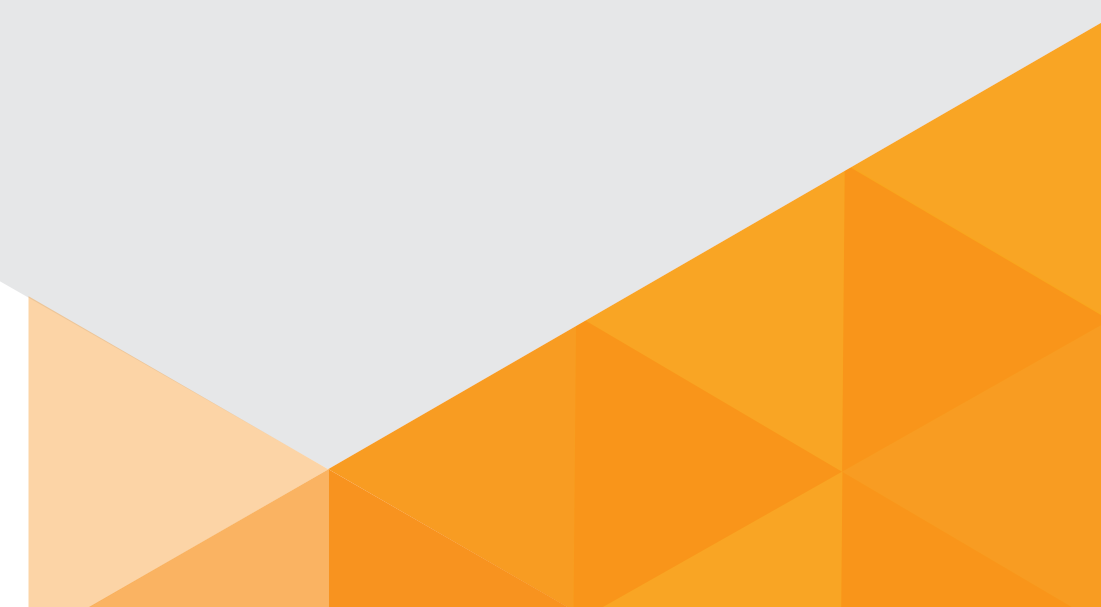

A lesão por pressão (LPP) deriva-se de um dano causado a pele e/ou tecidos moles subjacentes, que geralmente ocorre sobre proeminência óssea ou associada à utilização de dispositivos médicos e outros artefatos. Tal condição sucede à pressão intensa ou prolongada associada muitas vezes ao cisalhamento (ALVES et al., 2016). Leva-se em consideração, ainda, o estado nutricional do paciente, o microclima, as comorbidades envolvidas e a perfusão (ALMEIDA; CARVALHO, 2015).

No Brasil, estatisticamente existem poucos estudos sobre a prevalência e incidência do agravo, contudo, no que tange ao domicílio, estudos apontaram entre $41,2 \%$ e 59\% de risco para o desenvolvimento da lesão por pressão, e uma prevalência entre $8 \%$ e 23\%, sendo considerado fator preocupante por se tratar de um evento que pode ser prevenido em até 95\% dos casos, conforme determina a Declaração do Rio de Janeiro sobre a Prevenção da Lesão por Pressão (SOARES et al., 2018).

Dentre os fatores associados ao risco de desenvolvimento de LPP, destacam-se a hipertensão arterial sistêmica, diabetes, inconsciência, imobilização, perda de sensibilidade, perda de função motora, perda de continência urinária ou fecal, presença de espasmos musculares, deficiências nutricionais, anemias, índice de massa corporal muito alto ou muito baixo, doenças circulatórias, doença arterial periférica, imunodeficiência ou uso de corticosteroide e tabagismo (MORAES et al., 2016).

Pessoas com sensibilidade diminuída, imobilidade prolongada ou acamadas, com idade avançada são as mais susceptíveis à LP, que ocorrem mais frequentemente nas regiões mentoniana, occipital, escapular, cotovelo, sacral, ísquio, trocânter, crista ilíaca, joelho, maléolo e calcâneo (MAZZO et al., 2018).

Neste contexto, na busca pela redução do agravo, o enfermeiro como integrante da ESF tem papel fundamental no assistir com melhores práticas de cuidado, propiciando às pessoas e seus familiares se tornarem protagonistas no processo de autocuidado. No entanto, para evitar a lesão por pressão, além de medidas próprias, o enfermeiro necessita de uma avaliação sistemática, com instrumentos disponíveis no domínio internacional, como a Escala Preditiva de Braden, que vem apresentando altas taxas de sensibilidade e especificidades no Brasil (SOARES et al., 2018).

Para tal, este estudo teve como problemática: "Quais evidências científicas apontam a eficácia da utilização da escala de Braden como ferramenta de avaliação de fatores de risco de lesão por pressão em idosos?" Para responder tal questionamento o estudo tem como objetivo principal avaliar na produção científica a eficácia da escala de Braden para avaliação de fatores de risco de lesão por pressão em idosos. 
O presente estudo trata-se de uma pesquisa bibliográfica do tipo revisão integrativa da literatura. Este procedimento foi escolhido por possibilitar a síntese e análise do conhecimento científico já produzido sobre o tema "UTILIZAÇÃO DA ESCALA DE BRADEN COMO FERRAMENTA PARA AVALIAÇÃO DE FATORES DE RISCO DE LESÃO POR PRESSÃO EM IDOSOS". Esta revisão utilizou a metodologia proposta no estudo de Oliveira et al. (2016).

De acordo com Ercole, Melo e Alcoforado (2014), a revisão integrativa de literatura é um método que tem como finalidade sintetizar resultados obtidos em pesquisas de maneira sistemática, ordenada e abrangente, mediante diferentes metodologias. É denominada integrativa porque fornece informações mais amplas sobre um assunto, constituindo um corpo de conhecimento e podendo ser direcionada para a definição de conceitos, revisão de teorias ou análise metodológica dos estudos. Este método proporciona a combinação de dados da literatura teórica e empírica, proporcionando maior compreensão do tema de interesse. Sua elaboração está estruturada em seis etapas distintas apresentadas na figura 1.

Figura 1:Etapas de construção de uma revisão integrativa

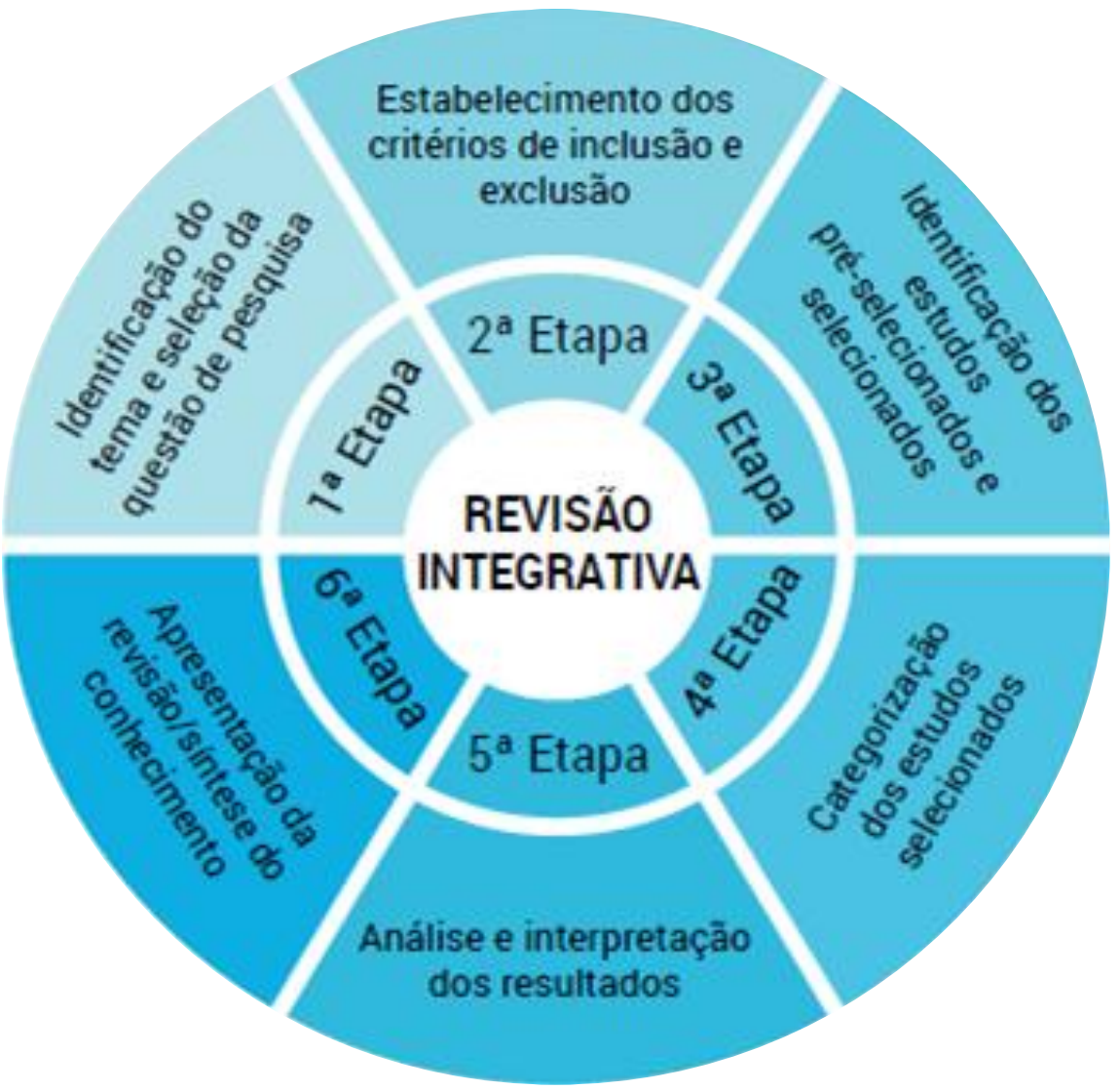

Fonte: Adaptado de Botelho; Cunha; Macedo, 2011, p.129. 


\section{IDENTIFICAÇÃO DO TEMA E SELEÇÃO DA QUESTÃO DE PESQUISA}

O tema "UTILIZAÇÃO DA ESCALA DE BRADEN COMO FERRAMENTA PARA AVALIAÇÃO DE FATORES DE RISCO DE LESÃO POR PRESSÃO EM IDODOS", determinou a construção da estratégia PICO, que representa um acrônimo para Paciente (P), Intervenção (I), Comparação (C) e Desfechos (O-outcomes), na qual foi utilizada para a geração da questão norteadora desta revisão integrativa da literatura: "Quais evidências científicas apontam a eficácia da utilização da escala de Braden como ferramenta de avaliação de fatores de risco de lesão por pressão em idosos?" Para a localização dos estudos relevantes, que respondessem à pergunta de pesquisa, utilizou-se de descritores indexados e não indexados (palavras-chave) nos idiomas português, inglês e espanhol. Os descritores foram obtidos a partir do Medical Subject Headings (MESH), dos Descritores em Ciências da Saúde (DeCS), como mostra o Quadro 1.

Consultou-se por meio de descritores e palavras-chave as bases de dados PubMed da National Library of Medicine; BVS (Biblioteca Virtual da Saúde), coordenada pela BIREME e composta de bases de dados bibliográficas produzidas pela Rede BVS, como LILACS, além da base de dados Medline e outros tipos de fontes de informação.

Quadro 1: Elementos da estratégia PICO, descritores e palavras-chave utilizados - Caxias, MA, Brasil, 2019.

\begin{tabular}{|c|c|c|c|c|}
\hline & ELEMENTOS & Mesh & Decs & Palavras-chave \\
\hline $\mathbf{P}$ & "Idosos" & "Aged" & $\begin{array}{l}\text { "Aged" } \\
\text { “Idosos" } \\
\text { "Anciano" }\end{array}$ & $\begin{array}{l}\text { "Aged" } \\
\text { “Idosos" } \\
\text { "Anciano" }\end{array}$ \\
\hline I & "Escala de Braden" & "scales" & $\begin{array}{l}\text { "Scales" } \\
\text { "Báscula" } \\
\text { "Escalas" }\end{array}$ & $\begin{array}{l}\text { “Scales" } \\
\text { "Báscula" } \\
\text { "Escalas" }\end{array}$ \\
\hline C & - & - & - & - \\
\hline 0 & "Lesão por Pressão" & "Pressure Ulcer" & $\begin{array}{l}\text { "Pressure Ulcer" } \\
\text { "Úlcera por Presión" } \\
\text { "Lesão por Pressão" }\end{array}$ & $\begin{array}{l}\text { “Wounds" } \\
\text { "Heridas" } \\
\text { "Ferimentos" }\end{array}$ \\
\hline
\end{tabular}

Fonte: Descritores, Títulos e Palavras-chaves.

O elemento $C$ da estratégia PICO não foi abordado nesta pesquisa pois esta não tem por objetivo comparar intervenções. Os termos utilizados durante a pesquisa foram classificados e combinados nos bancos de dados, resultando em estratégias específicas de cada base. 
Quadro 2 - Estratégias de busca utilizadas nas bases de dados BIREME, PUBMED e CINAHL-Caxias, MA, Brasil, 2018.

\begin{tabular}{|c|c|c|c|c|}
\hline BASE DE DADOS & ESTRATÉGIA DE BUSCA & 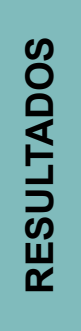 & 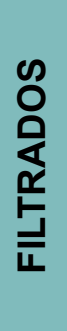 & 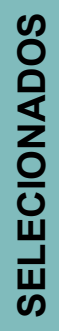 \\
\hline $\begin{array}{c}\text { BIREME } \\
\text { (descritores Decs) }\end{array}$ & (tw:(idosos)) AND (tw:(escala)) AND (tw:('lesao por pressao')) & 297 & 50 & 03 \\
\hline $\begin{array}{c}\text { PubMed } \\
\text { (descriptors MeSH) }\end{array}$ & ((aged) AND scales) AND 'pressure ulcer' & 168 & 9 & 1 \\
\hline
\end{tabular}

Fonte: Bases de dados.

\section{ESTABELECIMENTO DOS CRITÉRIOS DE INCLUSÃO E EX- CLUSÃO}

Como critérios de inclusão utilizaram-se estudos disponíveis em sua totalidade, publicados nos últimos cinco anos, de 2013 até 2018, nos idiomas Português, Espanhol e Inglês. Foram excluídos da busca inicial capítulos de livros, resumos, textos incompletos, teses, dissertações, monografias, relatos técnicos e outras formas de publicação que não artigos científicos completos.

\section{IDENTIFICAÇÃO DOS ESTUDOS PRÉ-SELECIONADOS E SELECIONADOS}

A análise para seleção dos estudos foi realizada em duas fases, a saber: Na primeira, os estudos foram pré-selecionados segundo os critérios de inclusão e exclusão e de acordo com a estratégia de funcionamento e busca de cada base de dados.

Encontrou-se duzentos e noventa e sete (297) estudos como busca geral na BVS, sendo que limitando a busca para artigos com texto completo realizado com humanos nos últimos cinco anos, obteve-se cinquenta (50) estudos, destes foram analisados títulos e resumos onde apenas três (3) estudos foram condizentes com a questão desta pesquisa.

Na base PUBMED, como busca total foram encontrados cento e sessenta e oito (168) estudos, aplicando na pesquisa o filtro que limita por texto completo dos últimos cinco anos com humanos, obteve-se nove (9) estudos, destes foram analisados títulos e resumos e teve como resultado final de um (1) estudos.

Na segunda fase os estudos foram analisados quanto ao potencial de participação no estudo, avaliando o atendimento à questão de pesquisa, bem como o tipo de investigação, objetivos, amostra, método, desfechos, resultados e conclusão, resultando em quatro (4) artigos.

Ao final quatro (4) artigos atenderam a questão norteadora e forma adicionados ao estudo. 
Figura 2 - Fluxograma do processo de seleção dos estudos para a revisão integrativa- Caxias, MA, Brasil, 2017.

\section{1a fase: Questão norteadora}

Quais evidências científicas apontam a eficácia da utilização da escala de Braden como ferramenta de avaliação de fatores de risco de lesão por pressão em idosos?

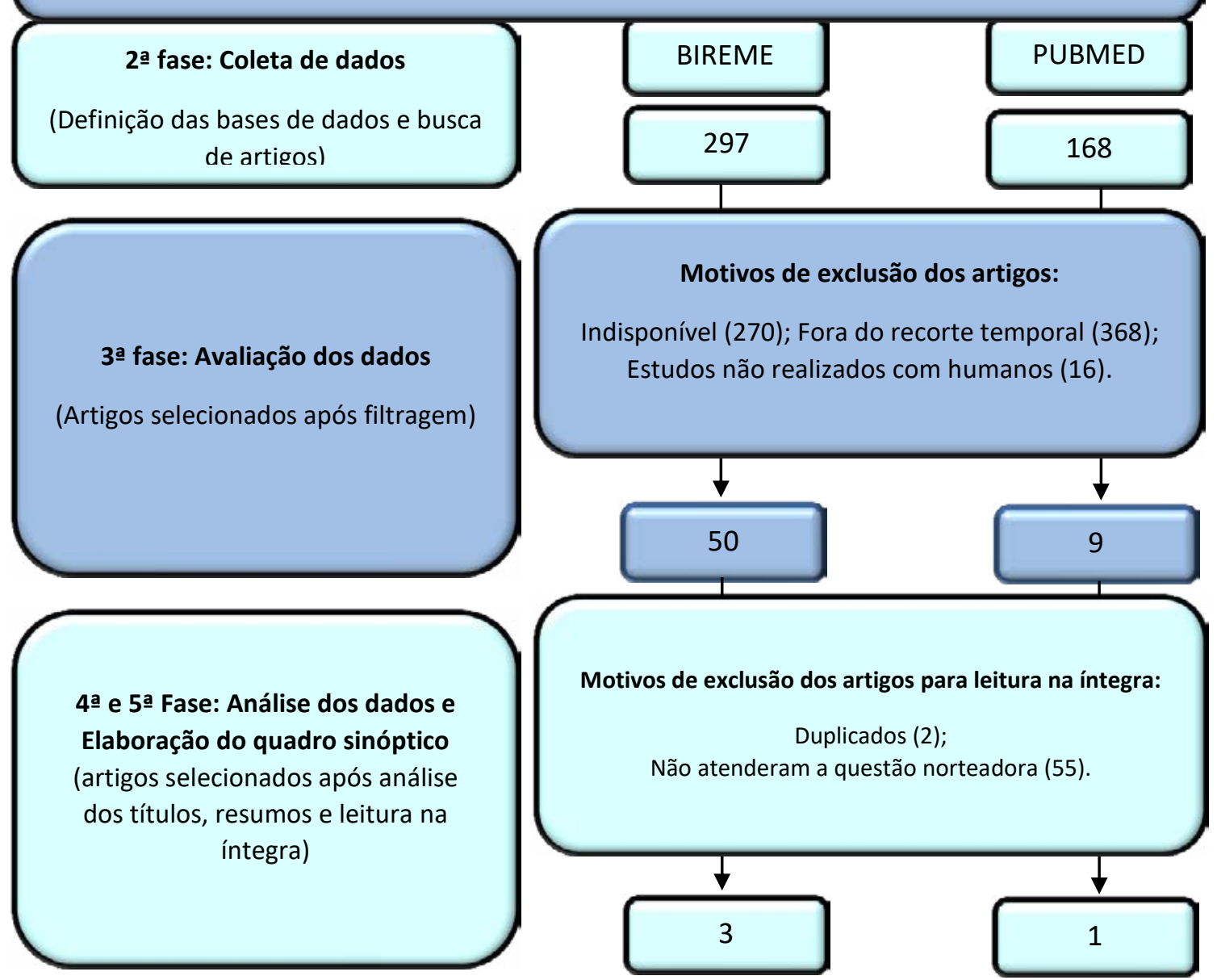

Fonte: Base de dados

\section{ANÁLISE E INTERPRETAÇÃO DOS RESULTADOS}

Nesta etapa foram analisadas as informações coletadas nos artigos científicos e criadas categorias analíticas que facilitou a ordenação e a sumarização de cada estudo. Essa categorização foi realizada de forma descritiva, indicando os dados mais relevantes para o estudo.

A pesquisa levou em consideração os aspectos éticos da pesquisa quanto às citações dos estudos, respeitando a autoria das ideias, os conceitos e as definições presentes nos artigos incluídos na revisão.

Optou-se pela análise em forma estatística e de forma de texto, utilizando cálculos matemáticos e inferências, que serão apresentados em quadros e tabelas para facilitar a visualização e compreensão.

As evidências científicas foram classificadas segundo os níveis e graus de recomendação propostos por Bork (2005), como mostra a figura 3 abaixo: 
Figura 2: Níveis de evidência e graus de recomendação
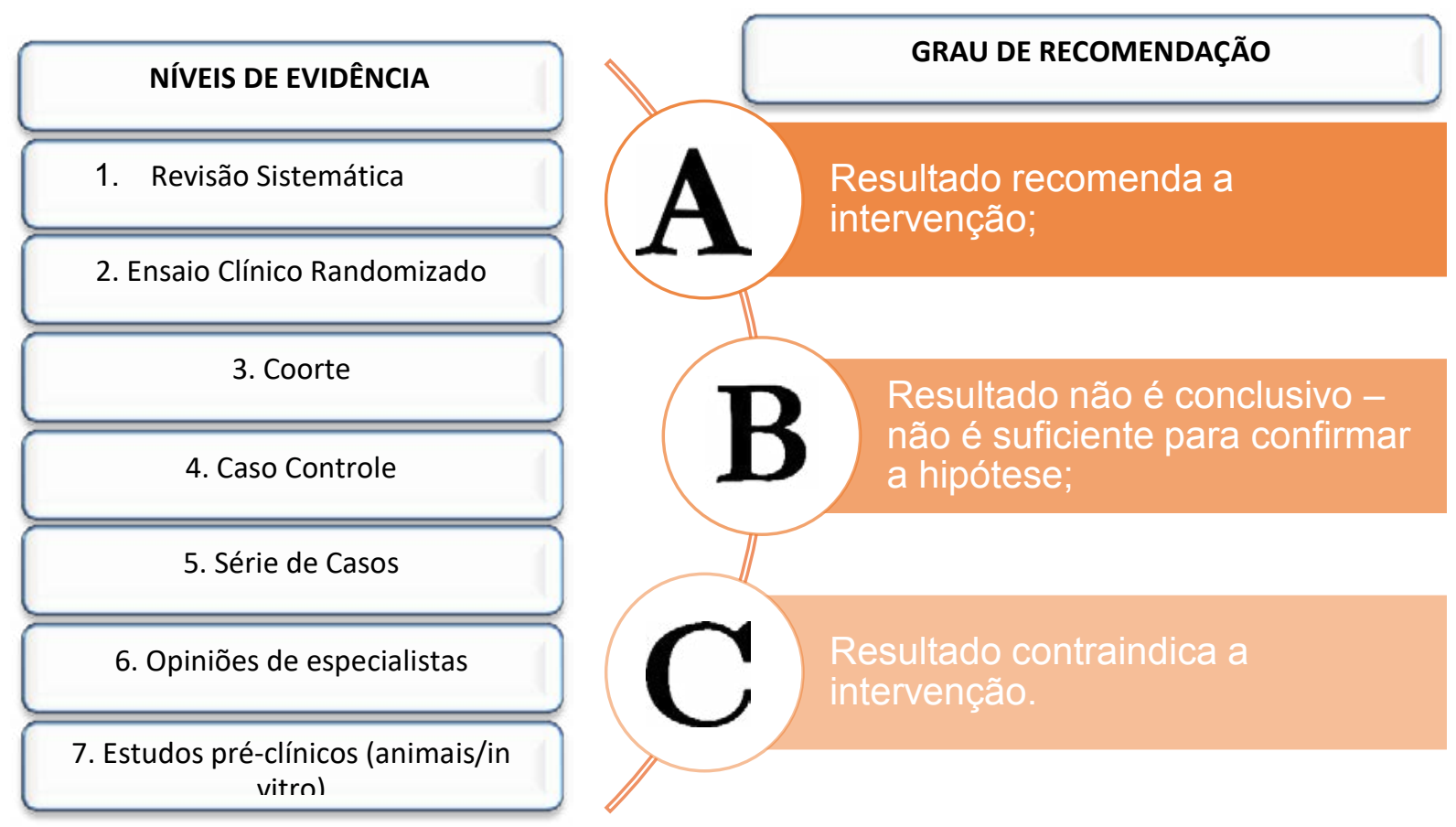

Fonte: base de dados (2005)

\section{RESULTADOS}

Dentre os quatro estudos incluídos nesta revisão, (2/50\%) estavam no idioma espanhol, (1/25\%) em português e (1/25\%) em inglês. A maioria das publicações foram concentradas no ano de 2014 (2/50\%). Em relação à natureza do estudo, houve prevalência de estudos de coorte (4/100\%). A principal linha de pesquisa investigada nessa temática versou sobre a confiabilidade da aplicação da escala de Braden na avaliação das úceras por pressão (Quadro 3). Os estudos avaliaram a eficácia da escala de Braden na detecção precoce de lesões por pressão(Quadro 4).

Quadro 3: Distribuição das publicações incluídas segundo o título, ano de publicação, país onde o estudo foi realizado, delineamento da pesquisa, nível de evidência e grau de recomendação. Caxias- MA, 2018.

\begin{tabular}{|c|c|c|c|c|c|c|c|}
\hline $\begin{array}{l}\text { № de } \\
\text { ordem }\end{array}$ & Autores & Titulo & $\begin{array}{c}\text { Base de dado } \\
\text { /ano de pu- } \\
\text { blicação }\end{array}$ & País & 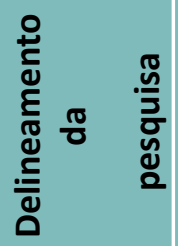 & $\frac{\frac{0}{0}}{\frac{\pi}{0}} \frac{\frac{\pi}{0}}{\frac{10}{0}}$ & 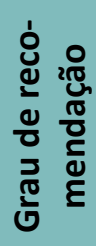 \\
\hline A.1 & $\begin{array}{l}\text { DÍAS et } \\
\text { al., } 2014\end{array}$ & $\begin{array}{l}\text { Fiabilidad de las escalas de } \\
\text { Braden y EMINA en pacientes } \\
\text { de atención domiciliaria inclui- } \\
\text { dos en programa de inmovi- } \\
\text { lizados / Reliability in Braden } \\
\text { and EMINA's scales for pa- } \\
\text { tients in domiciliary, included } \\
\text { in the immobilized patients' } \\
\text { programme }\end{array}$ & $\begin{array}{c}\text { BIRE- } \\
\text { ME/2014 }\end{array}$ & Espanha & $\begin{array}{l}\text { Estudo de } \\
\text { coorte }\end{array}$ & 3 & A \\
\hline
\end{tabular}


Valoración del riesgo de ulcera por presión según la escala

ASCAR- de Braden en el paciente neu-

A.2 RUZ et rológico / Assessing the risk

al., 2014 of pressure ulcers according

Braden scale in neurological patients

Aplicação da Escala de Braden em domicílio: incidência

FREITAS; e fatores associados a úlcera

A.3 ALBERTI, por pressão / Application of 2013

A.4

CHEN et

al., 2017

Construct Validity of the Braden Scale for Pressure Ulcer Assessment in Acute Care: A
BIRE-
ME/2014

Peru

Estudo de
coorte

3

A

\begin{tabular}{|c|c|c|c|c|}
$\begin{array}{c}\text { BIRE- } \\
\text { ME/2013 }\end{array}$ & Brasil & $\begin{array}{c}\text { Estudo de } \\
\text { coorte }\end{array}$ & 3 & A \\
\hline & & & \\
& & & \\
& & \\
\end{tabular}
Structural Equation Modeling

PUBMED/2017
Estudo de
coorte

Quadro 4: Publicações incluídas segundo objetivo principal, perfil amostral e principais resultados. Caxias, MA, 2018.

\begin{tabular}{|c|c|c|c|c|}
\hline Autores & $\begin{array}{l}\text { Objetivo Prin- } \\
\text { cipal }\end{array}$ & Perfil Amostral & Intervenções/interesse & Principais Resultados \\
\hline $\begin{array}{l}\text { DÍAS et } \\
\text { al.,2014 }\end{array}$ & $\begin{array}{l}\text { Verificar sua } \\
\text { confiabilidade } \\
\text { e comparar a } \\
\text { aplicabilidade de } \\
\text { ambas as escalas } \\
\text { nesta área, com } \\
\text { a qual teríamos } \\
\text { um instrumento } \\
\text { válido para a } \\
\text { medição do risco } \\
\text { de sofrer UPP em } \\
\text { todas as áreas da } \\
\text { saúde. }\end{array}$ & $\begin{array}{c}\text { Um total de } \\
30 \text { pacientes } \\
\text { foram incluídos } \\
\text { no estudo; } 62 \% \\
\text { eram mulheres } \\
\text { e } 38 \% \text { homens, } \\
\text { com uma idade } \\
\text { média de } 81 \\
\text { anos. }\end{array}$ & $\begin{array}{l}\text { Os dados foram coleta- } \\
\text { dos por profissionais de } \\
\text { enfermagem com uma } \\
\text { experiência média de } \\
\text { atendimento domiciliar } \\
\text { de } 20 \text { anos. Esses dados } \\
\text { foram coletados por meio } \\
\text { da observação direta so- } \\
\text { bre o estado da pele e o } \\
\text { restante por meio da en- } \\
\text { trevista com os pacientes } \\
\text { / cuidadores. }\end{array}$ & $\begin{array}{l}\text { De acordo com os resulta- } \\
\text { dos deste estudo, podisse } \\
\text { dizer que ambas as escalas, } \\
\text { definidas a partir de suas } \\
\text { respectivas sub-escalas são } \\
\text { confiáveis para medir o ris- } \\
\text { co de desenvolver UPP em } \\
\text { instrumentos de assistência } \\
\text { domiciliar, embora a consis- } \\
\text { tência interna dos itens foi } \\
\text { maior na escala Braden do } \\
\text { que na escala EMINA. }\end{array}$ \\
\hline $\begin{array}{c}\text { ASCAR- } \\
\text { RUZ et } \\
\text { al.,2014 }\end{array}$ & $\begin{array}{l}\text { Determinar o } \\
\text { risco de úlcera } \\
\text { por pressão no } \\
\text { paciente neuro- } \\
\text { lógico prostrado, } \\
\text { aplicando o Esca- } \\
\text { la de Braden no } \\
\text { Serviço de Neu- } \\
\text { rologia } 13 \text { A do } \\
\text { Hospital Nacional } \\
\text { Edgardo Reba- } \\
\text { gliati Martins. }\end{array}$ & $\begin{array}{l}\text { Foi determina- } \\
\text { do pela fórmula } \\
\text { da proporção } \\
\text { população finita } \\
\text { em que um ní- } \\
\text { vel de confiança } \\
\text { foi considera- } \\
\text { do } 95 \% \text { e um } \\
\text { erro de } 5 \% \text {. } \\
\text { Resultando } 75 \\
\text { pacientes sele- } \\
\text { cionados com a } \\
\text { tabela de núme- } \\
\text { ros aleatório. }\end{array}$ & $\begin{array}{l}\text { Para a coleta de dados a } \\
\text { observação foi utilizada. } \\
\text { Os instrumentos utiliza- } \\
\text { dos foram os de coletas } \\
\text { de dados e a escala de } \\
\text { Braden. Para coleta de da- } \\
\text { dos, foram planejadas as } \\
\text { seguintes etapas: a valida- } \\
\text { ção do instrumento como } \\
\text { um teste piloto, com um } \\
\text { total de dez pacientes } \\
\text { para determinar a confia- } \\
\text { bilidade e funcionalidade. }\end{array}$ & $\begin{array}{l}\text { Conclui-se que a escala de } \\
\text { Braden permite avaliar as } \\
\text { condições de fatores de } \\
\text { risco (percepção sensorial, } \\
\text { umidade, nível de atividade, } \\
\text { mobilidade nutricional) para } \\
\text { úlceras de pressão, especial- } \\
\text { mente em pacientes com } \\
\text { problemas neurológicos } \\
\text { como os do estudo. }\end{array}$ \\
\hline
\end{tabular}




\begin{tabular}{|c|c|c|c|c|}
\hline $\begin{array}{l}\text { FREITAS } \\
\text { et al.,2013 }\end{array}$ & $\begin{array}{l}\text { Estimar a capa- } \\
\text { cidade da Escala } \\
\text { de Braden em } \\
\text { predizer o surgi- } \\
\text { mento de úlcera } \\
\text { por pressão no } \\
\text { âmbito domi- } \\
\text { ciliar, conhecer } \\
\text { a incidência da } \\
\text { úlcera por pres- } \\
\text { são neste grupo } \\
\text { e os seus fatores } \\
\text { associados. }\end{array}$ & $\begin{array}{l}\text { Foram realiza- } \\
\text { dos com } 183 \\
\text { pacientes. Fi- } \\
\text { zeram parte da } \\
\text { casuística } 126 \\
(68,9 \%) \text { pa- } \\
\text { cientes do sexo } \\
\text { feminino e } 57 \\
(31,1 \%) \text { do sexo } \\
\text { masculino. Não } \\
\text { houve relação } \\
\text { estatística entre } \\
\text { o sexo e o sur- } \\
\text { gimento da UP } \\
(p=0,83) .\end{array}$ & $\begin{array}{l}\text { A Escala de Braden foi } \\
\text { aplicada em cada visita } \\
\text { domiciliária durante a } \\
\text { coleta de dados que se } \\
\text { estendeu no período de } \\
\text { janeiro a junho de } 2012\end{array}$ & $\begin{array}{l}\text { A Escala de Braden demons- } \\
\text { trou ser efetiva no âmbito } \\
\text { domiciliar para predizer os } \\
\text { pacientes com maior risco } \\
\text { de desenvolver úlceras por } \\
\text { pressão. }\end{array}$ \\
\hline $\begin{array}{l}\text { CHEN et } \\
\text { al., } 2017\end{array}$ & $\begin{array}{l}\text { Investigar a vali- } \\
\text { dade de constru- } \\
\text { to das escalas de } \\
\text { Braden usando } \\
\text { MEV. }\end{array}$ & $\begin{array}{l}\text { Este estudo foi } \\
\text { conduzido em } \\
\text { um hospital } \\
\text { universitário de } \\
3000 \text { leitos. A } \\
\text { cada ano, apro- } \\
\text { ximadamente } \\
2000 \text { a } 3000 \\
\text { pacientes com } \\
\text { risco de de- } \\
\text { senvolvimento } \\
\text { de úlceras por } \\
\text { pressão são } \\
\text { tratados nos } \\
\text { departamentos } \\
\text { de UTI e neuro- } \\
\text { logia, geriatria, } \\
\text { cirurgia cardía- } \\
\text { ca, neurocirur- } \\
\text { gia e ortopedia. }\end{array}$ & $\begin{array}{l}\text { Os registros médicos } \\
\text { foram revistos retrospec- } \\
\text { tivamente para caracte- } \\
\text { rísticas demográficas (ou } \\
\text { seja, pacientes idade, } \\
\text { sexo, peso e espectro da } \\
\text { doença) e escores total } \\
\text { e subescala de Braden. } \\
\text { Se mais de um escore de } \\
\text { Braden foi anotado, o me- } \\
\text { nor escore foi utilizado. }\end{array}$ & $\begin{array}{l}\text { O modelo original de Braden } \\
\text { Scale foi suportado por } \chi 2 \\
(9)=22,854, \mathrm{CFI}=0,902, \\
\text { GFI }=0,974 \text {, erro médio } \\
\text { quadrático de aproximação } \\
\text { (RMSEA) = 0,092, indican- } \\
\text { do ajuste inadequado do } \\
\text { modelo. Após modificação } \\
\text { de acordo com índices de } \\
\text { software, } \chi 2(2)=2,052, \mathrm{CFI} \\
=0,999, \mathrm{GFI}=0,999, \mathrm{RMSEA} \\
=0,020 \text { indicaram um ajuste } \\
\text { aceitável do modelo (mode- } \\
\text { lo final). As cargas fatoriais } \\
\text { de } 6 \text { subescalas foram todas } \\
\text { significativas (P <0,001), } \\
\text { com } 0,177 \text { para nutrição, } \\
137,3 \text { para atividade, } 0,167 \\
\text { para atrito e cisalhamento, } \\
825 \text { para percepção senso- } \\
\text { rial, } 0,626 \text { para mobilidade } \\
\text { e } 0,656 \text { para umidade sub- } \\
\text {-escala. }\end{array}$ \\
\hline
\end{tabular}

Os estudos abordaram sobre a utilização da escala de Braden na avaliação de risco de lesões por pressão (LPP). A maioria dos estudos teve como enfoque principal o cuidado das lesões por pressão através de escalas de predição (DÍAS et al., 2014; FREITAS; ALBERTI, 2013), eficácia e validade da escala de Braden no ambiente domiciliar (FREITAS; ALBERTI, 2013; DÍAS et al., 2014; VARGAS et al., 2014), fatores de risco para LPP e as categorias avaliadas na escala de Braden (CHEN et al., 2017; FREITAS; ALBERTI, 2013). 


\section{O CUIDADO DAS LESÕES POR PRESSÃO ATRAVÉS DE ES- CALAS DE PREDIÇÃO}

As LPP têm repercussões no sistema de saúde, nos próprios pacientes e em seus cuidadores, e representam um importante problema de saúde pública devido ao elevado número de casos. Um dos indicadores estabelecidos como um avaliador da qualidade dos cuidados de enfermagem é a LPP, como é referenciado no Plano de Qualidade para o Serviço Nacional de Saúde (2006-2010), no qual se propõe: "Melhorar a segurança dos pacientes em centros de saúde", e "prevenir a LPP em pacientes de risco" (DÍAS et al., 2014).

As escalas de predição de lesões têm se mostrado importantes para identificar pacientes de risco, permitindo direcionar os cuidados. Dentre os indicadores de validação de uma escala, têm-se: sensibilidade: mede a proporção de verdadeiros positivos entre os casos; especificidade: mede a proporção de verdadeiros negativos entre os controles; valor preditor positivo (VPP): mede a proporção de verdadeiros positivos entre os expostos; valor preditor negativo (VPN): mede a proporção de verdadeiros negativos entre os não expostos (FREITAS; ALBERTI, 2013).

\section{EFICÁCIA E VALIDADE DA ESCALA DE BRADEN NO AMBI- ENTE DOMICILIAR}

Días et al. (2014) relata que, a escala de Braden é considerada válida e eficaz para avaliar o risco de desenvolver LPP; além disso, essa escala é superior ao julgamento clínico na predição desse risco e, portanto, recomenda-se que as instituições incluam esta escala em seus protocolos. Em relação à capacidade preditiva e aos dados referentes à validação a escala de Braden apresenta resultado satisfatório no contexto hospitalar e sócio-sanitário, no entanto, mais estudos de validação serão necessários, especialmente no cenário da assistência domiciliar, onde ainda não foi validado.

O Programa de Acompanhamento Domiciliar se caracteriza por diminuir o índice de internação hospitalar, realizar procedimentos simples e atividades preventivas no domicílio, oferecendo maior conforto ao paciente e diminuição do custo para os convênios (VARGAS et al., 2014). De acordo com Freitas e Alberti (2013), a incidência de lesão por pressão no acompanhamento domiciliar é de 20\% e a escala de Braden demonstrou ser efetiva no âmbito domiciliar para predizer os pacientes com maior risco de desenvolvê-las.

\section{FATORES DE RISCO PARA LPP E AS CATEGORIAS AVALIA- DAS NA ESCALA DE BRADEN}

O principal fator de risco é a pressão por contato, quando a pressão é maior que $32 \mathrm{mmHg}$ para arteríolas e $12 \mathrm{mmHg}$ para vênulas por período prolongado impede o fluxo sanguíneo adequado, reduz a nutrição no local e propicia a deterioração tissular. Outro fator de risco é a força de cisalhamento, ocorre quando o paciente permanece imóvel na cama, enquanto as camadas da pele movimentam-se, sendo comum em pacientes posicionados com a cabeceira muito elevada acima de $30 \circ$. Além dos fatores externos como riscos para o desenvolvimento de lesão por pressão, existem também os fatores internos relacionados como desnutrição, anemia e doenças crônicas (FREITAS; ALBERTI, 2013). 
A Escala de Braden para avaliação do risco de lesões por pressão é composta por 6 subescalas: percepção sensorial, umidade da pele, atividade, mobilidade, nutrição e fricção e cisalhamento. As cinco primeiras subescalas são pontuadas de um (menos favorável) a quatro (mais favorável); a sexta subescala, fricção e cisalhamento, é pontuada de um a três. A somatória total está entre os valores de seis a vinte e três. A contagem de pontos baixa, indica uma baixa habilidade funcional, estando com alto risco de o paciente desenvolver LPP (CHEN et al., 2017).

\section{BUNDLES DE INTERVENÇÕES}

Elencou-se um conjunto de duas intervenções de enfermagem necessárias para a prevenção de lesões por pressão (LPP) através da escala de Braden organizados com seus respectivos níveis de evidencia, podem ser visualizados, no quadro 5.

Quadro 5: Bundles de Intervenções para a aplicação da escala de Braden em pacientes com risco de LPP. Caxias-MA, 2018.

\section{INTERVENÇÕES}

Executar analise dos fatores de risco para lesões por pressão com escala de Braden

Aplicar reforço aos resultados da escala de Braden, com a escala de Emina

\section{NÍVEL DE EVIDÊNCIA}

III

III

\section{DISCUSSÃO}

Existe uma necessidade de estabelecer prioridades para as ações de prevenção de eventos adversos. A adoção de boas práticas referentes à execução de procedimentos deve ser priorizada. Portanto ao analisar os resultados dos estudos, os pesquisadores escolheram 2 (duas) intervenções para compor o bundle.

Desta forma, o bundle constitui-se pelas seguintes intervenções e níveis de evidência I e V: Executar analise dos fatores de risco para lesões por pressão com escala de Braden; Aplicar reforço aos resultados da escala de Braden, com a escala de Emina.

\section{EXECUTAR ANALISE DOS FATORES DE RISCO PARA LESÕES POR PRESSÃO COM ESCALA DE BRADEN}

Em uso há mais de 30 anos, a Escala de Braden foi submetida a sugestões de revisão. Nas últimas duas décadas, muitos métodos de mineração de dados têm sido usados com sucesso para prever o risco com grande sucesso. A Escala de Braden para avaliação do risco de úlcera por pressão é composta por 6 sub-escalas: percepção sensorial, umidade da pele, atividade, mobilidade, nutrição e fricção e cisalhamento (CHEN et al., 2017).

Em termos de validade, 1 meta-análise 5 de 9 publicações revelaram que a sensibilidade combinada, especificidade, valor preditivo positivo e valores preditivos negativos foram de $86 \%, 38 \%, 28 \%$ e 93\%, respectivamente, no cuidado de longo prazo. Estes resultados demonstraram que a Escala de Braden teve validade na predição do risco de úlcera por pressão que foi melhor que o julgamento de enfermagem (CHEN et al., 2017). 


\section{APLICAR REFORÇO AOS RESULTADOS DA ESCALA DE BRADEN, COM A ESCALA DE EMINA.}

Escala validada por estudos anteriores em cuidados hospitalares e sua pontuação varia de 0 a 15; é uma escala operacional direta, ou seja, uma pontuação mais alta, um risco maior e uma definição operativa clara, ou seja, os itens são perfeitamente especificados, o que evita a variabilidade Inter observador. Essa escala também é dividida em várias sub-escalas, cujas pontuações são as seguintes: para a subescala "Estado Mental", de 0 a 3; para a subescala "Mobilidade", de 0 a 3; para a subescala "Incontinência húmida", de 0 a 3; para a subescala "Nutrição", de 0 a 3, e para a subescala "Atividade", de 0 a 3 (DÍAS et al.,2014).

Escalas BRADEN e EMINA são escalas contínuas que indicam o nível de risco de UPP (úlceras por pressão) pelos valores obtidos, e é inverso do outro, ou seja, quanto maior o valor observado da escala EMINA aumento do risco de UPP, enquanto Maior valor observado da escala de BRADEN diminuiu o risco de UPP. Dependendo das respostas obtidas para cada um dos itens que formam escalas, pode se fazer uma outra classificação dos pacientes em quatro possíveis subgrupos de risco: nenhum risco, baixo risco, médio risco e alto risco, de acordo com a pontuação das Escalas de ENIMA e BRADEN. (DÍAS et al.,2014). 


\section{CONCLUSÃO}

A escala de Braden foi identificada como um instrumento confiável e efetivo para prever os pacientes com maior risco de desenvolver leões por pressão. Dentre os efeitos demonstrados destacam-se os fatores de risco para lesão, as formas de prevenção da mesma, e a permeabilidade e eficácia da escala até mesmo em ambientes domiciliares.

As intervenções bundles trazem medidas que versam sobre a avaliação inicial e antecipada do desenvolvimento de lesão por pressão na pele de pacientes idosos, além do esclarecimento do modo de funcionamento das escalas de análise de lesão. 


\section{REFERÊNCIAS}

CHEN, H. L. et al. Validade de Constructo da Escala de Braden para Avaliação de Úlcera por Pressão em Cuidados Agudos: Uma Abordagem de Modelagem de Equações Estruturais. 2017. Disponível em: <https://www.ncbi.nlm.nih.gov/pubmed/28267683> Acesso em: 22 nov. 2018, 23:12:11.

DÍAS, F. J. G. et al. Fiabilidad de las escalas de Braden y EMINA en pacientes de atención domiciliaria incluidos en programa de inmovilizados. Gerokomos vol.25. 2014. Disponível em: < http://scielo.isciii.es/ scielo.php?script=sci_arttext\&pid=S1134-928X2014000300008> Acesso em: 20 nov. 2018, 12:34:00.

FREITAS, J. P. C. ALBERTI, L. R. Aplicação da Escala de Braden em domicílio: incidência e fatores associados a úlcera por pressão. 2013. Disponível em: <http://www.scielo.br/pdf/ape/v26n6/02.pdf> Acesso em: 21 nov. 2018, 23:55:00.

MAZZO, A. et al. Ensino de prevenção e tratamento de lesão por pressão utilizando simulação. Rio de Janeiro. 2018. Disponível em: <http://www.scielo.br/scielo.php?pid=\$1414=81452018000100701-\&scriptsci_arttext\&tlng=pt> Acesso em: 24 nov. 2018, 12:12:00.

OLIVEIRA, F. B. M. et al. Relação entre a sobrecarga de trabalho e erros de administração de medicação na assistência hospitalar. Revista Ciências \& Saberes. Caxias, v.2, n.2. Out/Dez.2016. Disponível em: <http://www.facema.edu.br/ojs/index.php/ReOnFacema/article/view/212/81> Acesso em: 20 nov. 2017.

SOARES, C. F. HEIDEMANN, I. T. S. B. Promoção da saúde e prevenção da lesão por pressão: expectativas do enfermeiro da atenção primária. enferm. vol.27 Florianópolis. 2018. Disponível em: <http:// www.scielo.br/scielo.php?script=sci_arttext\&pid=S0104-07072018000200301> Acesso em: 23 nov. 2018, 11:20:00.

VARGAS, C. A. et al. Valoración del riesgo de ulcera por presión según la escala de Braden en el paciente neurológico. rev. Enfermeria Herediana. Vol. 7. 2014. Disponível em: <http://www.upch.edu.pe/ vrinve/dugic/revistas/index.php/RENH/article/view/2119> Acesso em: 20 nov. 2018, 20:11:00. 